\title{
Moralisieren ist kein Informationsersatz: zum Rollenverständnis des Journalisten
}

\author{
von Hermann Boventer
}

Wieso kann bevorzugter Gegenstand der Kommunikation der Kommunikator selbst werden? Der Kölner Soziologe Erwin K. Scheuch antwortet auf diese Frage: „Der Kommunikator hält sich selbst für eine Verkörperung des eigentlich Objektiven." Die sogenannten Kritischen unter den Journalisten hätten eine Wahrheit mitzuteilen, eben nicht mehr nur Bericht zu erstatten, bessere Einsicht zu vermitteln, Zweifel anzumelden. Der Funktion nach werde hier eine Priesterrolle beschrieben, meint Scheuch ${ }^{1}$.

Ein neues, journalistisches Priestertum? Diese Kritik ist hart, und die Intellektuellen, die sich als Journalisten oder Schriftsteller eine kritische Erzieherrolle zusprechen, bekommen es neuerdings zu spüren, was Tendenzwende heißt. Helmut Schelsky argumentiert in seinem jüngsten Werk nicht weniger scharf gegen die journalistische „Kaste der Sinnvermittler“. Sein Buch „Die Arbeit tun die anderen. Klassenkampf und Priesterherrschaft der Intellektuellen"2 nimmt den ideologischen Machtanspruch solcher Männer wie Rudolf Augstein, Günter Gaus, Alexander Mitscherlich oder Heinrich Böll ins Fadenkreuz. Es sind Menschen, die nach Joseph Schumpeters Urteil die Macht des geschriebenen und gesprochenen Wortes handhaben, dabei keine Kenntnisse aus erster Hand besitzen und sich durch eine kritische Haltung auszeichnen, die aus der Situation der Intellektuellen als Zuschauer und Außenseiter abgeleitet wird ${ }^{3}$.

In der Bundesrepublik sind in den letzten Jahren die emanzipatorischen Forderungen nach Gesellschaftsveränderung sehr stark in das journalistische Rollenverständnis eingebrochen. Eine Zunahme des Meinungs- und Kampfjournalismus ist zu beobachten. Auf den Mainzer Tagen der Fernsehkritik war die Rede von den „televisionären Zuchtmeistern", die ihren Beruf zunächst als politischen begriffen und es als ihre eigentliche journalistische Aufgabe ansähen, "gesellschaftliches Bewußtsein zu verändern“, den Angehörigen dieser Gesellschaft „immer wieder Denkanstöße zu versetzen “ oder, mit einem Wort, den Zuschauer zu erziehen ${ }^{4}$. Die kritische Theorie der Frankfurter Schule ist wiederzuerkennen, etwa Jürgen Habermas mit seinen Thesen, „daß die Uberlieferungsmuster auch anders sein könnten“, daß die „unmittelbare Aufstörung kultureller Selbstverständlichkeiten “ durch „einen universalen Rechtfertigungszwang" aller Normen heute verpflichtend sei. Habermas spricht zu den Journalisten das verräterische Wort, die Kommunikation dürfe nicht zur Information verkommen ${ }^{5}$.

Wind die Information unter der Herrschaft des subjektiven Emanzipationsjournalismus fortan notleidend sein? Der kontinentaleuropäische Journalismus hat sich mit der Kategorie der Objektivität niemals so recht befreunden können. „Objective journalism " ist im angelsächsischen Kulturbereich eine durchgehende Tradition geblieben, den Auswüchsen der amerikanischen oder englischen Sensationspresse

Dr. Hermann Boventer ist Direktor der Thomas-Morus-Akademie in Bensberg und Vorsitzender der Gesellschaft Katholischer Publizisten Deutschlands e. V. 
zum Trotz. Ideologisch scheint der objektive Journalismus weniger anfechtbar als der subjektiv-kämpferische. Er hat sich eine fast naive Aufmerksamkeit für das eigene Berufsethos erhalten können. Die Vereinigten Staaten mit ihren Journalistenschulen an den Universitäten liefern dafür manches Beispiel. Eines davon soll geschildert werden, bevor die Frage nach aktuellen Veränderungen im Rollenverständnis des Journalisten in der Bundesrepublik erneut aufgegriffen wird.

\section{Hippokratischer Eid für Journalisten}

Ein paar Autostunden westlich von St. Louis hat die amerikanische Staatsuniversität von Missouri ihren größten Campus. Die Stadt heißt Columbia. Hier befindet sich die älteste Journalistenschule der Vereinigten Staaten, die "Columbia School of Journalism". Sie hat sich durch ihre jährlichen Journalistenpreise — „for distinguished service in journalism" - einen internationalen Ruf erworben. In Amerika selbst hat ein Student mit einem Graduiertendiplom von Columbia eine journalistische Karriere so gut wie in der Tasche.

Die glückliche Verbindung von Theorie und Praxis ist das Auffallendste für einen deutschen Besucher. Dabei sticht besonders ins Auge, in welchem Maße die Zeitungsund Kommunikationswissenschaft eine wertende ist. Die Berufsethik hat einen hohen Stellenwert im Programm. Wie der Journalist sich selbst versteht und seine Arbeit einschätzt, wird am Objekt eingeübt. Die Stadt Columbia wird seit 1908 in ununterbrochener Folge mit einer Tageszeitung versorgt, die als "The Missourian" von den Studenten in Gemeinschaftsredaktion mit den Professoren der Journalistenschule hergestellt wird. Als Laboratorium dienen auch eine universitätseigene Radiostation und ein Fernsehstudio mit Regionalprogramm.

Dem Bulletin der Schule ist eine Art von Hippokratischem Eid für Journalisten vorangestellt, "The Journalist's Creed“, in dem manches recht dick aufgetragen ist, aber mit jenem freimütigen Glauben an das Gute, den sich Amerika trotz innerer Niederlagen bis heute erhalten hat: "Ich glaube, daß klares Denken und klares Sprechen, Genauigkeit und Fairneß grundlegend sind für einen guten Journalismus. Idh glaube, daß ein Journalist nur schreiben soll, was er in seinem Herzen für wahr hält..." Solchen Glaubensbekenntnissen begegnet man oft in Amerika. Feierlich eingerahmt wie eine Magna Charta mit Brief und Siegel, hängen sie als Wandspruch auf dem Flur des journalistischen Seminars, in der Eingangshalle, im Redaktionsbüro: "Ich glaube, niemand sollte als Journalist schreiben, was er nicht sagen würde als Gentleman."

Glückliches Missouri, woher hast du deine Kriterien? Allerdings irrt sich der Besucher, wollte er solche Tafeln, die alle mit „I believe“ beginnen, nur als Wandsprüche abtun. Die Erziehung zur Wertung des eigenen Handwerks ist integrierender Bestandteil der Ausbildung. In einer Vorlesungsklasse, die gerade die Frage erörtert, wie objektiv der Journalist schreibt und welche Schwierigkeiten er im Umgang mit der Wahrheit hat, wird diese Beobachtung bestätigt.

Der Journalismus sieht sich in zwei Hauptrichtungen eingeteilt. Demnach gibt es einen objektiven Journalismus, der die Welt reflektiert und sie nicht zu verändern sucht. Dieser Journalismus ist neutral, so weit das möglich ist. Er ist leidenschaftslos und saugt die Ereignisse wie ein Schwamm in sich auf, sie möglichst unvoreingenommen weiterzuvermitteln. Die Informationspublizistik steht ganz im Vordergrund. 
Die unbedingte Trennung von Nachricht und Kommentar wird aufrechterhalten.

Diesem Typ des Journalismus wird ein zweiter gegenübergestellt, sieht man von Mischformen ab. Er wird als propagandistischer, subjektiver Journalismus gekennzeichnet. Der engagierte Journalist ist angesprochen, der sich eine Führungsrolle zuspricht und auf das Bewußtsein der Gesellschaft einzuwirken versucht. Dieser Journalist betrachtet sich als Anwalt der Menschheitswerte. Sein Konzept ist nicht marktorientiert, sondern elitär.

\section{Kommunikationsethik als Aschenbrödel}

Was uns hier als Lehrmeinung gegenübertritt, klassifiziert den Journalismus nur unvollkommen. Dieses Gebilde ist zu komplex für eine reine Typenlehre. Anhand der aufgewiesenen Merkmale lassen sich dennoch gewisse Trends diagnostizieren auch für die geistige Situation des Journalismus und seiner produzierenden Trägergruppen in der Bundesrepublik. Das soll im folgenden geschehen. Unsere These lautet, daß der engagierte, subjektive Meinungs- und Kampf journalismus die letzten Jahre zugenommen hat bei uns. Das Rollenverständnis des Journalisten verändert sich. Die Entwicklung verläuft in Richtung auf erzieherische, propagandistische und advokatorische Wirkungen. Das geht zu Lasten der Information und der Ausgewogenheit des Meinungsklimas. Der Journalist wird zum Parteigänger, die Information zur polit-pädagogischen Waffe im Meinungsstreit.

Inwieweit solche Veränderungen der ausübenden Trägergruppe selbst bewußt sind, müßte durch Untersuchungen erhärtet werden. Die Philosophie der Kommunikation scheint in Deutschland aus ihrer Aschenbrödel-Rolle schwer herauszufinden. Entsprechend ist auch die Berufs- und Kommunikationsethik vernachlässigt. Nach dem Dritten Reich und den Erfahrungen mit dem Goebbels'schen Propagandaministerium war die Skepsis gegenïber jeglichem Anspruch an die neugewonnene Pressefreiheit nicht ungeredhtfertigt; zu lange war man gegängelt worden. Heute arbeiten die Journalisten in der Bundesrepublik so frei wie in kaum einem anderen Land. Solange sich Traditionen und Verhaltensmuster in ihrem Handwerk erhalten konnten, die einen tragenden Grund für das praktische Handeln abgaben, war die Berufsethik sozusagen mitgliefert worden. Damit ist es vorbei. Normen, Spielregeln und

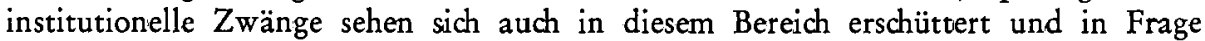
gestellt. Mit dem Nachwuchs kommen zunehmend engagierte Veränderer, Propagandisten und Erzieher in die Redaktionen.

Haben wir uns einzurichten auf einen neuen und entschieden-engagierten Typ von Journalismus, der sein Ethos aus dem Gesinnungsraum nimmt und sich nicht als Informations-, sondern vorrangig als Meinungs-und Erziehungsjournalismus versteht?

\section{Maßstäbe des objektiven Journalismus}

Der objektive Jornalismus hat seine geistige Heimat im liberal-angelsächsischen Kulturraum und ist in Deutschland nie heimisch geworden. James Reston von der "New York Times“ sieht die Anfänge des objektiven Journalismus mit dem Aufkommen drahtloser Agenturen zusammenfallen. Associated Press und United Press hatten eine zunehmend große Zahl von Zeitungen mit Nachrichtenmaterial zu beliefern, das neutral sein mußte wegen der heterogenen Kundschaft. Dieser 
Trend zur Objektivität sah sich verstärkt durch die Pressekonzentration, die in USA viel früher eingetreten ist als bei uns. Zum Beispiel wird eine Zeitung, die ein Lokalmonopol erringt und einen heterogenen Leserkreis bedient, objektiver berichten als ein kämpferisches Gesinnungsblatt, das mit dem Konkurrenten ständig im Clinch gelegen hat.

Neuerdings hat die Medienpolitik diese Frage auch für die Bundesrepublik aufgeworfen. Der Zeitung mit lokaler Alleinstellung wird monopolartiges Verhalten vorgeworfen. Es ist eine offene Frage, ob es etwa in einer Kreisstadt zu einem besseren Meinungsklima kommt, wenn zwei oder drei auflagenschwache, wirtschaftlich gefährdete Zeitungen miteinander im Wettbewerb stehen oder ein einziges, aber wirtschaftlich gesundes Blatt einen Pluralismus praktiziert, der ihm vom Markt aufgezwungen wird. Nun ist hierzu unlängst in einer Studie festgestellt worden, daß eine Verkürzung der publizistischen Leistung nach Erlangung des Lokalmonopols nicht ausfindig $\mathrm{zu}$ machen war. Diese Untersuchung wurde von Professor Elisabeth Noelle-Neumann vorgenommen, zusammen mit dem Allensbacher Meinungsforschungsinstitut und dem Institut für Publizistik der Mainzer Universität. Das Fazit erbrachte, daß Zeitungen mit lokaler Alleinstellung den entscheidenden Meinungsäußerungen ihre Schärfe nehmen, daß der Abdruck von Leserbriefen mit gegensätzlichen Standpunkten zunimmt, ebenso die Argumentationsbreite bei kontroversen Themen. In der Kontrollfunktion gegenüber öffentlichen Instanzen hätten Zeitungen mit Lokalmonopol keineswegs nachgelassen, meinte die Studie?.

Das Ergebnis ist nicht überraschend, weil mit größener Marktnähe der Wille zur Objektivität und Ausgewogenheit schon immer zugenommen hat. Das ist kein Plädoyer für das Lokalmonopol, sondern für eine differenzierte Betrachtungsweise

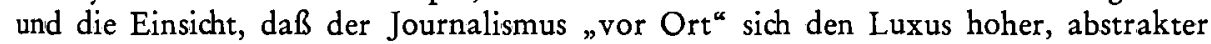
Gesinnungen am wenigsten leisten kann. Je geringer die Marktnähe - das gilt für die elektronischen Medien, auf die noch zurückzukommen ist - desto größer die Neigung, die sachliche Information durch Propaganda zu ersetzen.

\section{Tatsachen durch das menschliche Filter}

Kehren wir zurück zum objektiven Journalismus. Was zählt und den Ausschlag gibt, ist der ständige Versuch auf Seiten des Journalisten, möglichst objektiv und unvoreingenommen zu berichten. Das zeigt sich in der Sorgfalt seiner Recherchen und seines Vorgehens, auch in seinem Zögern, zu klassifizienen und zu schematisieren, wenn er sein Urteil mitteilt. Der objektive Journalist individualisiert seine Probleme, er weiß um ihre Komplexität und Unausschöpfbarkeit. Ereignisse und Personen sind unbegrenzt in der Vielfalt ihrer Möglichkeiten, und mit einer solchen Grundannahme eine Nachricht zu redigieren, ein Bild oder ein Urteil zu veröffentlichen, macht das journalistische Argumentieren schwieriger, aber konstruktiver.

Heute ist es modisch geworden, die Preisgabe objektiver Standards damit zu rechtfertigen, daß ein genereller Ideologieverdacht unterstellt wird. Der Journalismus, der sich an objektiven Werten orientiert, wird verdächtigt, seine Interessen für die Erhaltung des Bestehenden einzusetzen und unter dem Deckmantel der „Objektivität" eine handfeste Machtpolitik zu betreiben. Die Ideologieverdächtiger sind allerdings meistens auf einem Auge blind, nämlich dem linken, wo die Handfestigkeit wenig zu wünschen übrigläßt und die eigenen Interessen heiliggesprochen werden. 
Ein Journalismus, der seine Hauptaufgabe im Entlarven, Verdächtigen und fortwährenden Aufdecken von Mißständen sieht, wird sich selbst zur Ideologie. Er hat den Kontakt mit der menschlichen Realität verloren.

Festzuhalten bleibt, daß es eine objektive Information im kategorischen, idealen Sinn nicht gibt. Der Journalist ist ein Mensch, keine Maschine. Sämtliche Tatsachen und Ereignisse laufen durch das menschliche Filter. Der Manipulation sind technisch auch kaum noch Grenzen gesetzt. Das objektive Ethos kann sich nur in Annäherungswerten realisieren. Darauf kommt es jedoch entscheidend an, daß eine Grundhaltung vorliegt, die sich trotz subjektiver Bedingtheiten nicht von Forderungen der Sorgfalt, Vollständigkeit, Ausgewogenheit, Wahrhaftigkeit, Fairneß, Leidenschaftslosigkeit abbringen läßt, sei der Meinungsdruck auch noch so stark.

\section{Gesinnung und Propaganda}

Journalismus und Askese scheinen ein sehr ungleiches Paar, aber beide haben mehr miteinander zu tun, als es den Anschein hat. Ein Journalist, der nicht weglassen kann, der nicht schweigt, wo Schweigen angemessen ist, ein Journalist, der sich ständig selbst zu verwirklichen sucht und alles herausholt, was ihm einfällt und gefällt, wäre ein schlechter Journalist. Die geprägte Form, auch und vor allem die Sprachform als eigentliches Handwerkszeug des Journalisten verlangt immer Abstriche. Die Sprache will gemeißelt sein. Präzision zeigt sich in der knappen Darlegung, nicht im Schwafeln. Wer sich anbiedert, geht auch im Journalismus unter sein Niveau. Kühle und Gelassenheit sind angemessene Tugenden. Die distanzierenden unter den Tugenden stehen den Massenmedien am besten an.

Das aufklärerische Erbe des kontinentaleuropäischen Journalismus hat sich mit einer zurückhaltend-asketischen Auffassung nicht befreunden können. Die Zeitgenossen der Französischen Revolution preisen den Schriftsteller und Publizisten als „geborenen Advokaten der Menschheit". Ihm sei es vorbehalten, die Stimme der öffentlichen Gerechtigkeit in Bewegung zu setzen. Bei Joseph von Görres ist der publizistische Auftrag mit idealisch hohen Worten umschrieben: „Einer muß da sein, der da die Wahrheit zu sprechen verbunden ist, unumwunden, ohne Vorbehalt und Hindernis.... Die aber, die da kundtun die Meinung der Versammlung, seien Herolde, aus der Menge ausgewählt, als Männer bewährter Treue und geprüfter guter Gesinnung. " Nach einem anderen Wort von Görres ist Publizität „der mächtige Hebel, der das Geisterneich in Bewegung setzt. Den Bösewicht prangert sie unbarmherzig an. Ihr Falkenauge durchringt alle Nebel. "8

Bei Joseph von Görres verbindet sich der bürgerlich-aufklärerische Offentlichkeitsbegriff mit dem gesinnungsethischen, der in der Tradition der katholischen Kirche verhaftet ist ${ }^{9}$. Die neue liberale Pressefreiheit des 18. Jahrhunderts war in den Augen der Kirche eine "geduldete" Pressefreiheit. Im Mittelalter war der Offentlichkeitsanspruch der Kirche identisch mit dem Wahrheitsanspruch. Mit der Emanzipation des Individuums wurde dieser Anspruch relativiert und aufgehoben. Die Kirche konnte jedoch von ihrem Wahrheitsanspruch nicht abrücken. Jede Information blieb instrumentalisiert und in den Dienst größerer Ziele genommen. Sie hatte stets der Wahrheit zu dienen. Die Kirche hat für den zur wirkungsvollen Propaganda zugehörigen Apparat auch immer schon einen guten Sinn gezeigt. 1662 wurde das päpstliche Missionsinstitut "Congregatio de propaganda fide“ gegründet. 
Nach dem Etymologie-Duden hat Propaganda etwas zu tun mit "weiter ausbreiten, ausdehnen, durch Senkreis fortpflanzen“, auch mit "pfropfen“. Die Propaganda der Wahrheit war der Gegenstand des kirchlichen, später katholisch-publizistischen Bemühens, und in dieser Tradition steht auch Görres.

In dem Handbuch der Publizistik des 1969 verstorbenen Zeitungswissenschaftlers Emil Dovifat, der eine ganze Generation seiner Fachrichtung verkörpert, kommt das Stichwort "Objektivität" nur am Rande vor. Für einschlägige Hinweise muß man schon unter "Gesinnung" oder "Nachrichtenpolitik" nachschlagen. Bei den hauptsächlichen Erscheinungsformen hat Dovifat die verbreitende, erobernde Publizistik neben der kämpfenden, angreifenden, zerstörenden Publizistik aufgeführt. Eine Erscheinungsform, die der Information eigenen Rang zuspricht, kommt nicht vor. Der dynamische Wille, „zu beeinflussen, ja zu führen“, steht ganz im Vordergrund. Der gesinnungsethische Ansatz bestimmt die journalistische Grundhaltung. Der Journalist erscheint als ein Mann der innersten Überzeugung und des öffentlichen Bekenntnisses. "Gesinnung, freilich im Guten und im Bösen", ist nach Dovifat die Atmosphäre, „durch die jede publizistische Aktion sich darbietet. Es gibt keine Aktion, die nicht die gute Absicht, das ethische Ziel, den öffentlichen Dienst für sich in Anspruch nähme. " ${ }^{10}$

Die Wegstrecke von der Information zur bewußten Einflußnahme, "Propaganda“ und Gesinnungspublizistik ist sehr kurz in dem Dovifat'schen Porträt der journalistischen Persönlichkeit. Aristoteles wird zitiert, wonach der Publizist „in den Menschen das Glaubenerweckende wachzurufen" hat. Wie leicht dieser Ansatz in die totalitäre Publizistik umschlägt, war Dovifat wohl bewußt. Dem Zitat des Artikels 5 unseres Grundgesetzes setzt er die Verlautbarkeit des SEDPolitbüros vom 24. 4. 1959 gegenüber: „Als kollektiver Agitator, Propagandist und Organisator, hat jedes Publikationsorgan nicht nur das Denken zu beeinflussen und $\mathrm{zu}$ verändern, sondern Aktionen auf allen Gebieten der sozialistischen Umwälzung aufzulösen." Trotzdem gelangt Dovifat zu dem Schluß, die Nachrichtengabe vor allem auch durch die neuen publizistischen Mittel lasse ihn „kaum mehr an die Möglichkeit einer sachlichen Unterrichtung glauben "11.

Für die angelsächsische Maxime „Comments are free, but facts are sacred“ (Kommentare sind frei, aber Tatsachen heilig) zeigte dieser Zeitungswissenschaftler und Publizist wenig Neigung. Weimarer Republik und Drittes Reich hatten sein Gegenprogramm geprägt, ein Programm der kämpferischen Gesinnung, der Sittlichkeit des Wahren und Guten.

Heute sind die Ideologen in dieses Programm eingebrochen. Das begründet unsere Skepsis. Wir sehen den gesinnungethischen Humanitarismus groß auf die Fahne geschrieben, während hinter den Kulissen die geistige Machtergreifung stattfindet. Andererseits brauchen wir nicht so weit zu gehen wie Epiktets resignierender Satz „Sunt non res, sed rerum opiniones“, daß nämlich die Dinge nicht so erscheinen wie sie sind, sondern wie sie dargestellt werden. Dann würde Objektivität vollends zur Illusion. Man kann die erkenntnistheoretischen Zweifel auch so weit treiben. Kein Ethos, schon gar nicht das publizistische, kann in Reinform bestehen. Im praktischen Vollzug entscheiden die Intention und der Annäherungswert. Nimmt man die Kategorien der Gesinnungs- und Verantwortungsethik (Max Weber), wobei die letztere vor allem im politischen Handeln die angemessene ist, dann werden sich diese beiden Formen im journalistischen Handeln die Waage halten müssen. Daß 
der Journalismus der Wahrheit verpflichtet bleibt und dies oberster Grundsatz ist, hat der Deutsche Presserat noch unlängst in seinem dankenswerten Versuch einer Kodifizierung der journalistischen Pflichten erklärt ${ }^{12}$.

\section{Die Politisierung des Fernsehens}

Der Journalist muß sich fragen: Bin ich ein Propagandist? Oder liegt meine Aufgabe zunächst in der Vermittlung von Tatsachen und Informationen? Als der langjährige Monitor-Moderator Casdorff auf die Unvereinbarkeit von Ausgewogenheit und engagiertem Journalismus im Fernsehprogramm angesprochen wurde, antwortete er ohne Zögern: "Jawohl, wir wollen diese Gesellschaft verändern.“ Ein zeitkritisches Fernsehmagazin ist nach Casdorffs Meinung „betont subjektiv", und diese bewußte Meinungsäußerung sei vielleicht das wesentlichste Argument: "Das gilt nicht nur für die ganze Sendung, das gilt ganz besonders auch für den Moderator.... Er hat nach unserer Auffassung das Recht, seine sehr subjektive Meinung zu sagen ${ }^{13}$."

Der angezeigte Trend zum engagierten, subjektiven Journalismus hat sich seit einigen Jahren vor allem in den elektronischen Medien herausgebildet. Der Sparte der kritischen, politischen Magazine sind immer größere Teile der Sendezeit zuerkannt worden, in deutlicher Politisierung des Mediums und auf Kosten dokumentarischer, informatorischer oder unterhaltender Programmteile. Die Moderatoren-Rolle ist zum Prototyp dieses subjektiven Journalismus geworden. Damit straft der FernsehModerator seinen Namen Lügen, soll er doch moderieren, das heißt, ausgleichend und mäßigend beitragen, was an Fakten, Hintergründen, Meinungen und Wertungen zu einseitig gegeben wird oder überhaupt nicht, weil es nicht illustrierbar ist. Anstatt das Material für die Urteilsbildung der Zuschauer darzulegen, polarisiert und emotionalisiert der Moderator es durch seinen militanten Subjektivismus. Es entsteht eine positive Demagogie und somit ein Zerrbild der journalistischen Verantwortung. Wie soll es da noch zu einem redlichen Vermitteln kommen?

Im Fernsehen hat sich auch eine bestimmte Art von Nachrichtengebung eingebürgert. Das Medium "macht" seine Nachrichten selbst. Anhand des Ereignisses wird die Offentlichkeit "hergestellt". Partei A behauptet etwas durch ihren Sprecher, wozu Partei B etwas sagen muß, gesellschaftliche Gruppen gefragt sind, bis das gewünschte Meinungskonzert entsteht, das über die Wirklichkeit nichts, über höchst subjektive Meinungen nur Peripheres aussagt. Hier stehen dem Medium die eigenen Strukturen im Wege, wenn es sich selbst immer wieder zur billigen Unterhaltungsmaschine degradiert. So ganz Unrecht hat Marshall McLuhan nicht, wenn er behauptet: The medium is the massage. Wobei die Betonung darauf liegt, daß das Medium selbst die Botschaft (message) „ist“. Es fabriziert sie als Surrogat, als Pseudo-Information.

So wird das Fernsehen zur künstlerischen Meinungsfabrik. Was an Realitätsnähe fehlt, wird durch „Engagement “ ersetzt. Das Leiden daran, daß sein Wort die Wirklichkeit nicht bewegt, frustriert manchen Fernsehredakteur und läßt eine ideale Pflanzstätte für den Gesinnungsjournalismus entstehen. Der Zuschauer, nämlich der Mensch als das eigentliche "Objekt" der journalistischen Einwirkung, ist in Mondferne gerückt. Wo Wirklichkeit sich solchermaßen verflüchtigt, radikalisiert sich die Moral. "Halbgötter in Schwarzweiß und in Farbe, die man wieder auf die Erde herabholen müßte ${ }^{\alpha}$, tituliert Intendant Franz Barsig vom Sender Freies Berlin die Fernsehgewaltigen. Er bezeichnet es als eine "nicht zu übersehende Gefahr" 
unseres öffentlich-rechtlichen Rundfunksystems, daß „der Meinungsjournalismus, ja selbst der Kampfjournalismus" in den letzten Jahren erheblich an Boden gewonnen habe ${ }^{\mathbf{1 4}}$.

\section{Redakteure wollen selbst Politik machen}

Dem (der Intention und Rundfunkverfassung nach) objektiven Fernsehen folgt das subjektive einer engagierten Politisierung. Die Redakteure wollen selbst Politik machen. Die ausgewogene Sachlichkeit liefert nicht genügend Dramatik. Sie ist zu blaß, zu fad. Wir fragen angesichts einer solchen Entwicklung, deren Rad sich kaum noch zurückdrehen läßt: Ist der Journalist ein Politiker? Woher nimmt er sein Mandat zur politischen Einwirkung? Kurios ist, daß sich der kämpferische Journalismus heute nicht stärker im Zeitungsgewerbe etabliert, wo er im Meinungsstreit sich legitimieren könnte, sondern daß sich in der Bundesrepublik gerade unter dem Dach einiger öffentlich-rechtlicher Anstalten die Kräfte eines höchst streitbaren Journalismus sammeln, der seinen Eros in der Schock-Therapie erblickt. Der Zuschauer soll aus seinem behaglichen Dornröschenschlaf aufgerüttelt werden. Der Journalismus spricht sich einen emanzipatorischen Auftrag zu. Ist der Journalismus ein Erzieher?

Solche Fragen stellen, heißt nicht schon, sie eindeutig nach der einen oder anderen Seite beantworten wollen. Das Rollenverständnis des Journalisten ändert sich mit den Zeitgegebenheiten. Es ist nicht das erste Mal, daß der Journalist als Träger einer neuen Moral erscheint. Aufklärung und humanitäres Ethos machen ihn zum Advokaten der Menschheitswerte. Nach Gottfried Benn entsteht die Menschheit durch Propaganda. Mit Schriftstellern, Pädagogen, Künstlern, Literaten, Soziologen hat der Journalist gemeinsam, daß er sich als Intellektueller versteht. Mit ihnen hat er auch gemeinsam, daß ihm die Beschäftigung mit Ideen den Sinn für die Realitäten verstellen kann.

Den Hofnarr zu spielen, war schon immer die notwendige, tabuierte Rolle der Intellektuellen, die in Kritik und Angriff ihre einzigen Mittel zur Wirksamkeit sahen. Mit ihrem Einzug in die Massenmedien ist ihnen eine ungewohnte Verantwortung zugefallen, der viele nicht gewachsen sind. Wie sich eine gewisse öffentliche Kritik heute mit "vollem Herzen der Moral der anderen annimmt" und „in der Opposition des schlechthin unaufhörlichen Kritisierens“ ihr Ethos luxurieren läßt, das hat Arnold Gehlen in seinem Buch „Moral und Hypermoral "15 vorwiegend anthropologisch und in Kategorien einer gesteigerten Aggressivität als Folge des allgemeinen Traditionsverfalls, des Gelten- und Gewährenlassens interpretiert. Der moralisierenden Meinungskultur würde die biblische Geschichte mit dem Splitter und dem Balken recht gut anstehen, damit nicht demnächst auf allen Kanälen ein Savonarola die Fernsehzuschauer mit seiner "Gesellschaftskritik“ langweilt und der politisch interessierte Rest seufzend bei Courths-Mahler-Verfilmungen seine $\mathrm{Zu}$ flucht sucht.

\section{Das Advokatorische einer Journalisten-Demokratie}

Die Sprödigkeit gegenüber dem eigenen Berufsethos findet keine Entsprechung in den hohen Moralforderungen, die der subjektive Gesinnungsjournalist anderen gegenüber artikuliert. Die Radikalisierung der Moral, die alles und nur sich selbst nicht 
in Frage stellt, ist heute in starkem Maße eine publizistische Tat. Die Zunahme des advokatorischen Typs bedeutet für den Journalismus in unserem Land eine ernste Gefahr. Ein freies Informationswesen ist in seiner Substanz gefähndet, wenn das öffentliche Moralisieren zum Informationsersatz gemacht wird. Die Umkehr müßte von den Journalisten selbst kommen, in verstärkter Besinnung auf ihre Berufsethik und ihre Rolle im gesellschaftlichen Ganzen, die sich als Dienst am Menschen versteht.

Der Soziologe Scheuch behauptet, in modernen Gesellschaften sei das wichtigste Mittel zur Verringerung von Komplexität die Moralisierung von Sachproblemen. Von einer Story im "Spiegel" bleibe meist nicht sehr viel haften - mit einer wichtigen Ausnahme: Der Leser weiß, wer die gute Fee und wer der böse Zauberer ist ${ }^{10}$. Dennoch können wir auf das Kriterium der Informationszuverlässigkeit nicht verzichten, wie komplex die Zusammenhänge sich auch darstellen, solange wir ein freies Informationswesen als konstitutiv für unsere staatliche Ordnung ansehen.

Die eingangs erwähnte, Habermas'sche Abwertung der Information gegenüber der Kommunikation - Habermas spricht vom "herrschaftsfreien“ Diskurs und verweist damit dieses Konzept ins Reich der Utopie - ist ein ebenso untaugliches wie gefährliches Glasperlenspiel, das den sogenannten „Kritischen“ unter den engagierten Journalisten nur das Alibi ihrer Parteilichkeit liefert. Bis alle Glieder der Gesellschaft in der herrschaftsfreien Kommunikation die gemeinsam geschaute, offenbarte Wahrheit finden, vergeht noch eine gute Weile, und bis dahin sind wir auf solide, zuverlässige, umfassende Information angewiesen. Sie zuerst und nichts vor ihr bleibt das Handwerk des Journalisten und ist heute stärker denn je seiner Redlichkeit und Nüchternheit anheimgestellt.

Der moderne Mensch braucht in der Massengesellschaft die sachliche Information wie das tägliche Brot. Er braucht Orientierungs- und Verständigungshilfen. Der Informationsgrad des durchschnittlichen Lesers oder Zuschauers ist viel zu gering, als $\mathrm{daß}$ er ohne Hilfen, die ein ausgewogenes Bild des Für und Wider liefern, auskommen könnte. Erkennt der Rezipient nicht die Zusammenhänge, sondern wird er von vorneherein in die streitbare, einseitige Konfrontationsstellung manipuliert, ist Aggressivität oder unreflektierte Abwehr meistens die einzige Folge dieses auf unzulängliche Information gegründeten Urteils. Für einen solchen Menschen reduziert sich die Welt der Gegensätze, in der er lebt, zu einem abstoßenden Catch-as-catch-can. Auf eine stärkere Heraushebung des Dienstcharakters käme es an. Und darauf, daß der wahrheitsgemäßen und möglichst objektiven Information auch weiterhin die Priorität gebührt. Das Advokatorische einer Journalisten-Demokratie liegt nicht im Sinne des gemeinen Besten. „Konstruktiv, tolerant, aber niemals gleichgültig“, so definiert die Journalistenschule von Missouri den eigenen Kodex, „beherrscht, geduldig, stets voller Hochachtung für die Leser, aber auch stets furchtlos gegenüber allen Ungerechtigkeiten.... Das oberste Prinzip eines guten Journalismus ist das Maß seiner öffentlichen Dienstleistung. ${ }^{\star}$

Anmerkungen:

1. Erwin K. Scheuch, Kulturintelligenz als Machtfaktor - Intellektuelle zwischen Geist und Politik, Zürich 1974, S. 47.

2. Helmut Schelsky, Die Arbeit tun die anderen. Klassenkampf und Priesterherrschaft der Intellektuellen, Düsseldorf 1975. 
3. Josef Schumpeter, Kapitalismus, Sozialismus und Demokratie, 2. Aufl., Bern 1950, S. 237.

4. Bericht in "Die Welt" vom 2.11.1974, Die televisionären Zuchtmeister, von Dieter Meichsner.

5. Jürgen Habermas, Legitimationsproblem im Spätkapitalismus, Frankfurt 1973, S. 101, $194 .$.

6. Bulletin der University of Missouri-Columbia, School of Journalism, Vol 74, Nr. 21, Sept. 18, 1973, p. 2.

7. Vgl. FAZ v. 19.9.1974.

8. Zit. nach Emil Dovifat (Hrsg.), Handbuch der Publizistik, Bd. 1, Berlin 1968, S. 15.

9. Vgl. Hans Wagner, Das Ende der katholischen Presse, 1. Band, Ein notwendiges Übel, Aschaffenburg 1974, S. $16 \mathrm{ff}$.

10. Dovifat, a. a. O., S. 43.

11. Dovifat, a. a. O., S. 168 , S. 86 f.

12. Deutscher Presserat, Publizistische Grundsätze (Pressekodex), 12. 12. 1973.

13. Casdorff in einem Interview des Westdeutschen Fernsehens über "Monitor“ am 5.6.1973.

14. Vgl. Der Journalist, Heft 12, 1972, S. 28.

15. Arnold Gehlen, Moral und Hypermoral, Eine pluralistische Ethik, Bonn 1969, S. 152.

16. Scheuch, a. a. O., S. 38.

\section{S U M M A R Y}

In the Federal Republic of Germany, opinion and agressive journalism and pressure to change society is growing. Therefore, one has to ask, if information in the future is not going to be under subjective aspect. The author distinguishes between an objective journalism reflecting the world, but not necessarily changing them, and a more propagandistic subjective journalism trying to influence the conscience of society. The number of people from this subjective journalism is growing in editorial offices, but especially in the television stations, where they have come under an unhealthy political influence. The author asks for real orientation and guidance in the objective sense. Modern men in a mass society need factual and objective information, and only when this is made available to them, that it becomes truly a public service.

\section{RÉSUMÉ}

Selon l'opinion de l'auteur sont apparues ces dernières années, en République Fédérale d'Allemagne, des exigences émancipatrices pour une transformation de la société dans le sens de la compréhension journalistique des rôles. Le journalisme d'opinion et le journalisme de combat croissent. C'est pourquoi il demande si l'information ne souffrira pas, à l'avenir, d'un journalisme d'émancipation subjectif et prépondérant. Il fait la distinction entre un journalisme objectif qui reflète le monde et ne cherche pas à le transformer, et - outre les formes mixtes - un journalisme propagandiste et subjectif qui essaie d'influencer la conscience de la société. Avec la nouvelle génération augmente, dans les rédactions, le 
nombre des transformateurs engagés, des propagandistes et des éducateurs. Dans ce contexte, l'auteur attire surtout l'attention sur les offices de télévision dont les rédactions semblent, selon lui, tomber sous l'influence malsaine de la politisation. En opposition à cela, il intervient en faveur de véritables aides d'orientation et de compréhension. L'homme moderne, dit-il, a besoin, dans la société de masses, de l'information objective comme du pain quotidien. L'information véridique et autant que possible objective devient pour lui, dans ce sens-même, un service public.

\section{RES UMEN}

En la República Federal de Alemania surgieron durante los últimos años —-según el autor- - reivindicaciones emancipadoras, tendentes a conseguir un cambio social en lo que se refiere a la concepción del papel del periodista. Cobra vigor el periodismo combativo y adoctrinante. En este contexto el autor se pregunta si en el futuro la información misma no sufrirá a causa de un periodismo emancipado, eminentemente subjetivo. Dejando al margen formas mixtas, distingue entre periodismo objetivo, que reproduce la actualidad mundial tal como es y no pretende cambiar el mundo, y un periodismo subjetivo y propagandístico que trata de influir en la conciencia de la sociedad. Cada vez con más frecuencia participan en las redacciones reformadores, propagandistas y educadores. En este contexto el autor hace mención especial de las cadenas de televisión, cuyas redacciones - - opina_- se encuentran bajo la perniciosa influencia de 10 politizante. En contraposición propugna una verbabera ayuda a la comprensión y la orientación. En la sociedad de masas _-anade-_- el hombre moderno necesita la información objetiva tanto como el pan cotidiano. La fidelidad a la verdad y una información tan objetiva como sea posible constituyen - opina- el sentido del servicio público. 